\title{
CT Perfusion for Confirmation of Brain Death
}

\author{
J.J.S. Shankar and R. Vandorpe
}

\begin{abstract}
SUMMARY: For pronouncing brain death, unlike CTP, the 2-phase CTA gives no functional information and is limited by inadvertent delay of the second acquisition, which may give false-negative results. The purpose of our study was to compare CTP and CTA derived from the CTP data with the Dupas and Frampas criteria for confirmation of brain death. A retrospective review of CTP in 11 consecutive patients for confirmation of brain death showed a sensitivity of $72.7 \%$ for 7 - and 4 -point scores, $81.8 \%$ for opacification of the ICV, and $100 \%$ for CTP scores in the brain stem. CTA obtained from the CTP data showed similar sensitivity in the diagnosis of brain death. This protocol also reduces the iodinated contrast dose and is less operator-dependent. The addition of the functional tools of CTP increased the sensitivity of CTA in the confirmation of brain death.
\end{abstract}

ABBREVIATIONS: $A C A=$ anterior cerebral artery; ICU = intensive care unit; ICV = internal cerebral vein; STA = superficial temporal artery; VOG = great cerebral vein of Galen

$\mathrm{T}$ he diagnosis of brain death must comply with the medicolegal and ethical guidelines of each institution and should also be determined as accurately and as early as possible to avoid unnecessary treatment and allow organ harvesting for transplantation. Often, the diagnosis of brain death is made on the basis of clinical findings and neurologic examination. However for patients in the ICU with medication causing CNS depression, the clinical criteria of brain death, deep coma and the absence of brain stem reflexes, may be difficult to evaluate. In these cases, ancillary tests such as electroencephalography, cerebral angiography, nuclear scan, transcranial Doppler, CTA, and MR imaging/MRA are currently used in adults to pronounce brain death. ${ }^{1}$

Although cerebral angiography is considered the criterion standard, ${ }^{2-4}$ CTA has been accepted as one of the ancillary tests for confirmation of brain death. ${ }^{5,6}$ The protocol for CTA for the confirmation of brain death can vary considerably among different centers; this variation makes it difficult for it to be accepted as the criterion standard test. ${ }^{7}$ The most acceptable study, which used 2 acquisitions of CTA after a single bolus of contrast injection, had

Received April 30, 2012; accepted after revision August 26.

From Department of Diagnostic Imaging, Division of Neuroradiology, QE II Hospital, Halifax, Nova Scotia, Canada.

Paper previously presented at: 50th Annual Meeting of the American Society of Neuroradiology and the Foundation of the ASNR Symposium, April 21-26, 2012; New York, New York.

Please address correspondence to Jai Jai Shiva Shankar, DM, MSc, Department of Diagnostic Imaging, Division of Neuroradiology, QE II Hospital, Halifax, NS, Canada B3H1E6; e-mail: shivajai1@gmail.com, shivajai1@rediffmail.com

http://dx.doi.org/10.3174/ajnr.A3376 a variation between the 2 acquisitions from 54 to 61 seconds. ${ }^{5}$ Inadvertent further delay of the second acquisition may lead to diffusion of contrast into the distal cortical branches, causing false-negative results on CTA. Moreover CTA provides only anatomic and no functional information of the brain. The functional and the anatomic information about the brain can be obtained on CTP studies.

The purpose of our study was to compare the CTP and the CTA derived from the CTP data with the criteria of Dupas et $\mathrm{al}^{5}$ and Frampas et $\mathrm{al}^{6}$ for the confirmation of brain death.

\section{MATERIALS AND METHODS}

The data collected from July to December 2011 were retrospectively analyzed. The study was approved by our institutional ethics board.

\section{Patient Selection}

The definition of brain death in our ICU follows the Canadian guidelines. ${ }^{8}$ These include documentation of the etiology of coma; confounding factors precluding the diagnosis; bilateral absence of motor responses, excluding the spinal reflexes; and the core temperature of the patient. The absence of brain stem reflexes (which include bilateral absence of pupillary response to light, pupils equal to or greater than midsize, bilateral absence of corneal responses, bilateral absence of vestibulo-ocular responses, and absent gag and cough reflexes) is confirmed by 2 qualified physicians. Brain death is confirmed by ancillary testing when minimum clinical criteria cannot be completed or confounding 


\begin{tabular}{|c|c|c|c|c|c|c|c|c|c|c|c|c|c|c|c|}
\hline \multirow[b]{2}{*}{ No. } & \multirow[b]{2}{*}{$\begin{array}{l}\text { Age } \\
\text { (yr) }\end{array}$} & \multirow[b]{2}{*}{ Sex } & \multirow[b]{2}{*}{ Diagnosis } & \multicolumn{8}{|c|}{ CTA } & \multirow[b]{2}{*}{$C T P^{a}$} & \multirow[b]{2}{*}{$\begin{array}{l}\text { Delay } \\
\text { (h) }\end{array}$} & \multirow[b]{2}{*}{$\begin{array}{l}\text { 4-Point } \\
\text { Scale }\end{array}$} & \multirow[b]{2}{*}{$\begin{array}{l}\text { 7-Point } \\
\text { Scale }\end{array}$} \\
\hline & & & & STA & $\begin{array}{l}\text { RT } \\
\text { MCA }\end{array}$ & $\begin{array}{c}\mathrm{L} \\
\mathrm{MCA}\end{array}$ & $\begin{array}{c}\mathbf{R} \\
\mathrm{ACA}\end{array}$ & $\begin{array}{c}\mathrm{L} \\
\mathrm{ACA}\end{array}$ & $\begin{array}{c}\mathrm{R} \\
\mathrm{ICV}\end{array}$ & $\begin{array}{l}\mathrm{L} \\
\mathrm{ICV}\end{array}$ & VOG & & & & \\
\hline 1 & 46 & $\mathrm{~F}$ & SAH & $7 / 17$ & 1 & 1 & 1 & 1 & 1 & 1 & 1 & 1 & 1 & 1 & 1 \\
\hline 2 & 46 & $M$ & $\mathrm{ICH}$ & $6 / 16$ & 1 & 1 & 1 & 1 & 1 & 1 & 1 & 1 & 8 & 1 & 1 \\
\hline 3 & 63 & M & $\mathrm{ICH}$ & $9 / 17$ & 0 & 0 & 0 & 0 & 0 & 0 & 0 & 1 & 5.5 & 0 & 0 \\
\hline 4 & 48 & $M$ & $\mathrm{ICH}$ & $8 / 18$ & 1 & 1 & 1 & 1 & 1 & 1 & 1 & 1 & 9.5 & 1 & 1 \\
\hline 5 & 56 & $\mathrm{~F}$ & $\mathrm{SAH}, \mathrm{ICH}$, and IVH & $7 / 15$ & 1 & 1 & 1 & 1 & 1 & 1 & 1 & 1 & 1 & 1 & 1 \\
\hline 6 & 48 & M & $\mathrm{SDH}, \mathrm{ICH}$ & $8 / 22$ & 1 & 1 & 1 & 1 & 1 & 1 & 1 & 1 & 4.45 & 1 & 1 \\
\hline 7 & 57 & $\mathrm{~F}$ & $\mathrm{SAH}, \mathrm{IVH}$, and ICH & $8 / 16$ & 1 & 1 & 1 & 1 & 1 & 1 & 1 & 1 & 6.30 & 1 & 1 \\
\hline 8 & 68 & $\mathrm{~F}$ & $\begin{array}{l}\text { Collapsed after left occipital } \\
\text { nerve block }\end{array}$ & $8 / 22$ & 1 & 1 & 1 & 1 & 1 & 1 & 1 & 1 & 70 & 1 & 1 \\
\hline 9 & 52 & M & $\mathrm{SAH}, \mathrm{IVH}$, and ICH & $4 / 22$ & 1 & 1 & 1 & 1 & 1 & 1 & 1 & 1 & 3.15 & 1 & 1 \\
\hline 10 & 23 & M & $\mathrm{SAH}, \mathrm{ICH}$ & $8 / 22$ & 0 & 0 & 0 & 0 & 1 & 1 & 1 & 1 & 24 & 1 & 0 \\
\hline 11 & 23 & $\mathrm{~F}$ & $\begin{array}{l}\text { Hemorrhagic shock and } \\
\text { cardiac arrest }\end{array}$ & $6 / 21$ & 0 & 0 & 0 & 0 & 0 & 0 & 0 & 1 & 72 & 0 & 0 \\
\hline
\end{tabular}

Note:- L indicates left; R, right; ICH, intracerebral hemorrhage; IVH, intraventricular hemorrhage; SDH, subdural hemorrhage.

${ }^{a} \mathrm{CBF}$ - and CBV-matched defect in the brain stem.

b Time delay between CTA and the final declaration of brain death.

factors cannot be corrected. The confounding factors taken into consideration include unresuscitated shock; hypothermia (core temperature $<34^{\circ} \mathrm{C}$ ); severe metabolic disorders; peripheral nerve or muscle dysfunction or neuromuscular blockade; or dosing with anticonvulsants, sedatives, and analgesics. The standard ancillary test includes the demonstration of global absence of intracranial blood flow either by 4-vessel cerebral angiography or radionuclide cerebral blood flow imaging. CTA has been introduced and has almost replaced the 4-vessel cerebral angiography with time.

The prerequisite conditions that must be met before ancillary testing include the following: 1) established etiology, 2) deep unresponsive coma, and 3) absence of unresuscitated shock and hypothermia.

\section{Image Acquisition}

Instead of 2-phase CTA, all patients underwent a 9.6-cm-coverage brain CTP protocol $(80 \mathrm{kV}, 200 \mathrm{mAs}, 128 \times 0.6 \mathrm{~mm}$ collimation, 9.6-cm scan volume in the $\mathrm{z}$-axis by using an adaptive spiral scanning technique ["shuttle mode"], CT dose index of $189.64 \mathrm{mGy}$ ), with 18 scans every 1.67 seconds (inflow phase) followed by 4 scans every 3.47 seconds (outflow phase), resulting in a total scanning time of 40.17 seconds on the 128-section dual-energy CT scanner (Sensation Definition; Siemens Healthcare, Erlangen, Germany). A total of $40 \mathrm{~mL}$ of nonionic iodinated contrast media (iopamidol, Isovue-370; Bracco Diagnostic, Vaughan, Ontario, Canada) was injected at a rate of $5 \mathrm{~mL} / \mathrm{s}$, followed by a saline flush of $40-\mathrm{mL}$ sodium chloride at $5 \mathrm{~mL} / \mathrm{s}$ and a start delay of 5 seconds. Two sets of axial images with a section thickness of $1.5 \mathrm{~mm}$ for the CTA analysis and $5 \mathrm{~mm}$ for the perfusion analysis were reconstructed without overlap and sent to the PACS. Sagittal and coronal multiplanar and maximum-intensity-projection images from the CTA data were reconstructed and sent to the PACS.

Perfusion analysis was performed for patients in whom intracranial arteries were seen on the axial source images with the vendor-supplied Neuro-VPCT software (Siemens Healthcare), by using the semiautomatic deconvolution algorithm Auto Stroke. Motion correction and bone segmentation were per- formed automatically; automatic arterial and venous vessel identification, vessel segmentation threshold, and depiction of a healthy hemisphere for normalization were reviewed on all patient datasets by a single observer (J.J.S.S.). Gray-scale and colorcoded perfusion parameter maps for $\mathrm{CBF}, \mathrm{CBV}$, and TTP were stored in a DICOM format.

\section{Image Analysis}

The images were analyzed first for the appearance and disappearance of contrast media in the superficial temporal artery branches to the scalp to confirm the successful intravascular injection and complete coverage of a cardiac cycle, respectively. The presence of contrast in the different segments of the intracranial arteries was analyzed on CTA on a 4-point scale on the basis of lack of opacification of the cortical segments of the MCA and the 2 ICVs. ${ }^{6}$ The same dataset was then used to evaluate the 7-point scale on the basis of lack of opacification of the intracranial ICA, M4 segment of the MCA, the A2 segment of the ACA, the ICV, and the VOG. ${ }^{4}$ A score of 1 was given for each of the nonopacified vessel segments on the 22nd volume of the CTP images, which was acquired 47.1 seconds after the injection of the contrast media.

\section{CTP Score}

We propose a CTP score of 1 when there is a matched decrease in $\mathrm{CBF}$ and $\mathrm{CBV}$ in the brain stem. This is to draw a parallel to the clinical findings of the absence of brain stem reflexes.

\section{Statistics}

We calculated the sensitivity of each segment of the intracranial arteries and that of the 4- and 7-point scale for each patient, taking the clinical confirmation of brain death as the criterion standard. The sensitivity of CTP parameters in the brain stem was also calculated for each patient by a similar method.

\section{RESULTS}

In the 6-month interval, 11 patients, all of whom were clinically suspected of brain death, were referred to us for the CTA ancillary test for confirmation of brain death. The details of these patients are shown in the Table. Patient 2 was the only one who was re- 


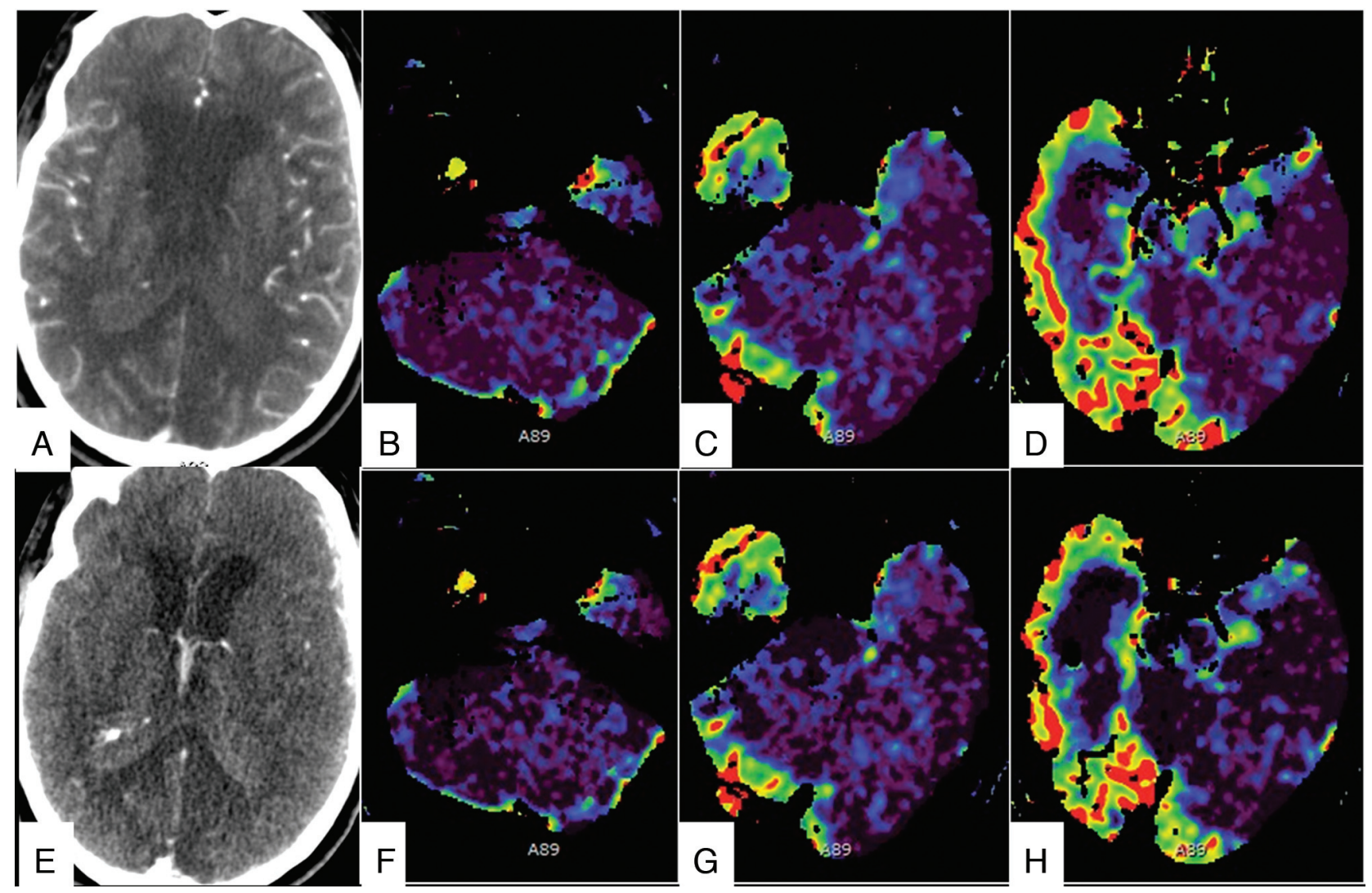

FIG 1. Different phases of CTA images in a 63-year-old man who had intracranial hemorrhage after tPA treatment for a thrombosed iliac stent show opacification of cortical branches of the MCA, A2 segment of the ACA (A), and the ICV and VOG (E). CTP images show a matched defect in the brain stem and cerebellum on $\mathrm{CBF}(B-D)$ and $\mathrm{CBV}(F-H)$ maps. The perfusion was preserved in the supratentorial compartment.

ferred during working hours and who underwent 3-phase radionuclide CBF imaging for brain death confirmation 12 minutes before undergoing our protocol. Patient 8 also had a second CTA acquisition performed 4 minutes after the CTP acquisition.

The opacification of different segments of the intracranial arteries is shown in the Table. Nine of the 11 patients were declared brain dead on our CTA protocol. Patients 3 and 11 had no perfusion in the brain stem but had preserved perfusion to the rest of the brain, and they were not declared brain dead but were suggested to have brain stem death (Fig 1). Both patients were subsequently pronounced dead 12 and 72 hours, respectively, after CTP.

\section{Vessel Opacification and CTA Score}

The appearance and disappearance of the contrast in the STA were seen during the acquisition of different volumes of CTP in all cases, suggestive of coverage of a complete cardiac cycle in all cases. The contrast appearance in the STA varied from the 4 th to the 9th volume, and the contrast disappearance varied from the 15 th to the 22 nd volume of CTP images.

In patient 8 , the second-phase CTA showed opacification of the intracranial ICA, M4 segment of the MCA, A2 segment of the ACA, the ICV, and the VOG. We believe that this was secondary to the diffusion of the contrast into these vessels with time.

\section{Seven-Point CTA Score}

Eight of the 11 patients had a CTA score of 7 (sensitivity of $72.7 \%$ ). A score of 3 was recorded in 1 patient who had opacification of the cortical branches of the MCA and the pericallosal arteries but no opacification of the ICV and VOG. A score of 0 was recorded in 2 patients who had opacification of the intracranial ICA, M4 segment of the MCA, A2 segment of the ACA, the ICV, and the VOG. One of these patients had opacification of the basilar artery, and the other did not.

\section{Four-Point CTA Score}

Eight of the 11 patients had a CTA score of 4 (sensitivity of $72.7 \%$ ). A score of 2 was recorded in 1 patient who had opacification of the cortical branches of the MCA and the pericallosal arteries but no opacification of the ICV. Therefore, the opacification of ICV was absent in $81.8 \%$ of patients. The same 2 patients had a score of 0 on 4-point and 7-point scoring systems.

\section{CTP Score}

All 11 patients showed no or matched decrease in CBF and CBV in the brain stem (sensitivity of $100 \%$ ). Nine patients did not show any $\mathrm{CBF}$ in the brain stem, and 2 patients (patients 3 and 11) showed matched decreases in CBF and CBV in the brain stem, suggestive of brain stem death (Figs 1 and 2). In both of these patients, there was preserved CBF and CBV in the supratentorial 


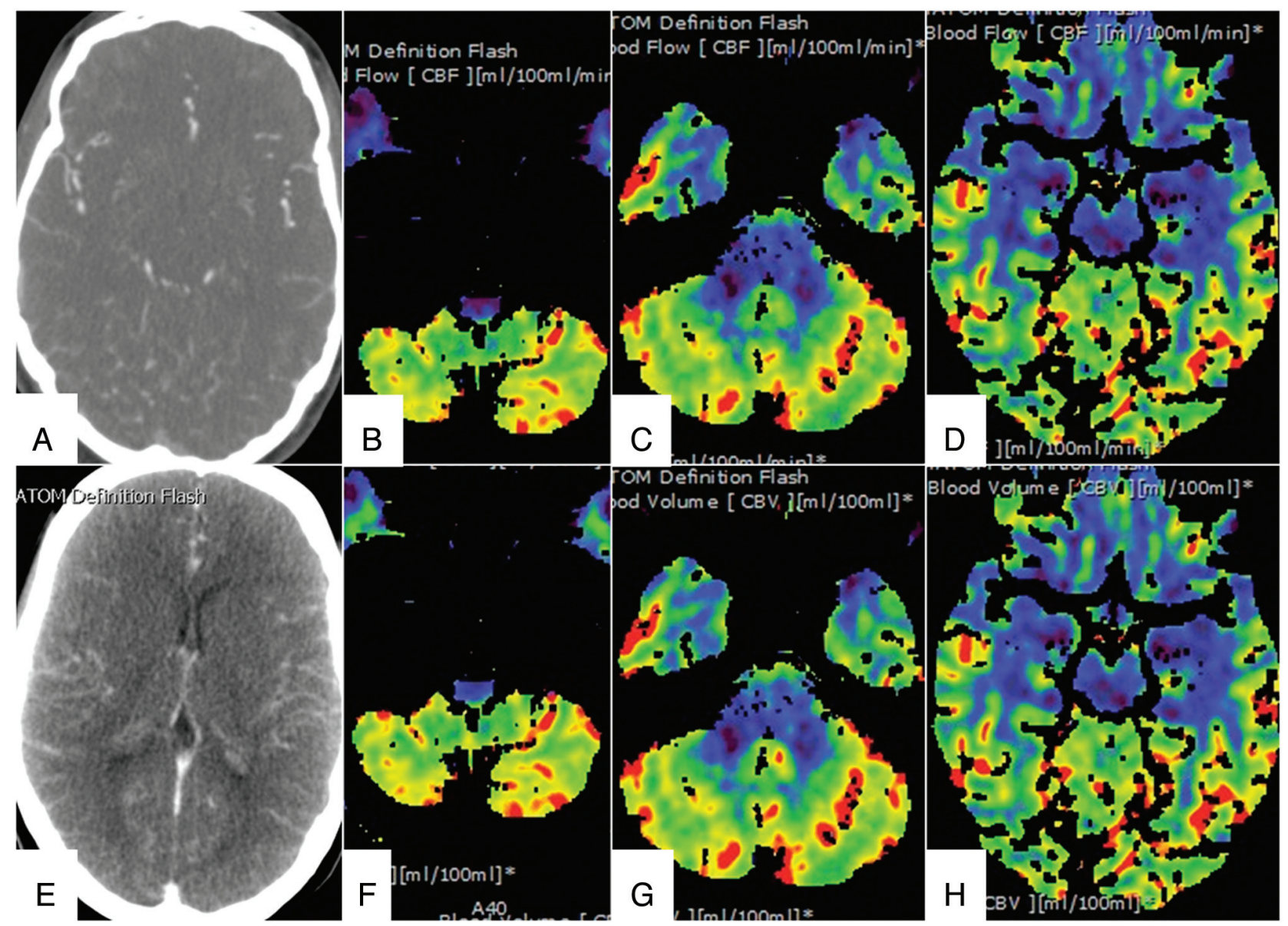

FIG 2. Different phases of CTA images in a 23-year-old woman who had hemorrhagic shock and cardiac arrest following a perforated innominate vein during central line insertion show opacification of the cortical branches of the MCA, A2 segment of the ACA $(A)$, and the ICV and VOG (E). CTP images show a matched defect only in the brain stem on CBF $(B-D)$ and $C B V(F-H)$ maps. The perfusion was preserved in the cerebellum and supratentorial compartment.

compartment, and 1 of these 2 patients had preserved CBF and CBV in the cerebellum (patient 11) (Fig 2).

\section{DISCUSSION}

Severe brain injury is a prerequisite for brain death, which, in turn, is a prerequisite for cadaveric organ donation. The demonstration of lack of cerebral circulation, required as an ancillary test, can be used when uncertainty exists about the reliability of parts of the neurologic examination or when the apnea test cannot be performed. In some protocols, ancillary tests are used to shorten the duration of the observation period. These ancillary tests have a potential for false-positives (ie, the test suggests brain death but the patient does not meet clinical criteria). Ideally these tests should be highly sensitive and specific, noninvasive, widely available, and operator-independent. Conventional cerebral angiography is considered the criterion standard because it is highly sensitive. However, it is invasive and time-consuming and requires an experienced neuroradiologist; these conditions can delay the examination. More recently, 2-phase CTA has emerged as an acceptable noninvasive alternative for conventional cerebral angiography ${ }^{5}$ because CTA is more widely available and can be relatively less operator-dependent. However, there are still limitations to CTA in the diagnosis of brain death. ${ }^{9}$ We believe these limitations are mainly due to a nonstandardized protocol and the need for multiple acquisitions. The 2-phase CTA still has a component of operator dependence. If the second phase is delayed for some reason, the diffusion of contrast in the distal arteries can give false-negative results.

Moreover, CTA gives only anatomic information without any functional information. The addition of the functional test of CTP eliminates both limitations and makes the test much more reliable for accurate confirmation of brain death. Clinical confirmation of brain death requires only the testing of the brain stem function. We find this an important disparity in the ancillary testing, in which demonstration of global absence (instead of only absence of CBF to brain stem) of intracranial blood flow is required. This disparity was possibly due to the lack of a tool that could selectively measure the $\mathrm{CBF}$ of the brain stem in the past. However, with more experience with CTP, we believe that this may serve as a tool to measure global brain stem function. The declaration of death was delayed by $>24$ hours in our study in the patients in whom brain death could not be confirmed on the basis of the CTA criteria, though there was evidence of brain stem death on CTP. Both of these patients were finally declared dead on the basis of the clinical criteria 24-72 hours after CTA. The importance of functional information in the determination of brain death is highlighted by our patient with brain stem death because 
these patients cannot perform any vital functions of the body without a viable brain stem. These patients possibly have shown false-negativity in previous studies. A similar case was described by Escudero et al. ${ }^{10}$

The CTA in our study showed similar sensitivity on a 7-point scale but lower sensitivity on a 4-point scale compared with the study of Frampas et al. ${ }^{6}$ The lower sensitivity of the 4-point scale can be explained simply by a smaller number of patients in our study. Opacification of the ICV was absent in $81.8 \%$ of patients. Most interesting, the CTP score in the brain stem was $100 \%$ sensitive. Our small study proposes that CTP may become a powerful ancillary tool for an early declaration of brain death. However, this needs to be validated in a larger number of patients.

CTP like CTA is a noninvasive and widely available tool. With the availability of automated software, CTP is relatively operatorindependent. The time-resolved CTA derived from the CTP data can be used for the anatomic information. The sequential volume acquisition of the brain while the contrast is being injected obviates a second acquisition. This also eliminates any chance of falsenegativity due to potential delay in the second acquisition.

Many patients being evaluated for brain death are potential organ donors, including kidneys. Most transplanted kidneys show evidence of acute tubular necrosis. This can be further exacerbated by the injection of a larger volume of potentially nephrotoxic iodinated contrast media for CTA. Our protocol uses only one-third of the volume of contrast media, to give similar if not better information. We believe this may help in reducing renal damage in the donor kidneys.

The higher radiation dose of CTP can potentially be a limitation of our protocol. However, in these terminally ill patients, the radiation dose may not be a vital issue. Moreover, the radiation dose can be further reduced by using the modified protocol suggested by Shankar et al. ${ }^{11}$

Variation in the quantitative analysis by using a different postprocessing method is a potential limitation of using CTP. The solution for this is to have normative data for every institution. Moreover, for diagnosis of brain death, we need only qualitative analysis rather than quantitative analysis. Limited coverage of the brain on CTP has been a concern in the past, but with the increased coverage provided by newer multisection CT scanners, this is no longer a limitation. ${ }^{12}$ With time, our limited experience with CTP will increase. The CTP score may not be very useful in conditions that affect the brain stem without resulting in brain death and may show lower CBV and CBF. It is difficult to imagine such a clinical situation, but in these cases, other clinical circumstances of the patient will have to be correlated with the CTP findings.

\section{CONCLUSIONS}

The CTA obtained from the CTP data showed similar sensitivity compared with the criteria of Dupas et $\mathrm{al}^{5}$ and Frampas et $\mathrm{al}^{5}$ in the diagnosis of brain death. This protocol also reduces the iodinated contrast dose and is less operator-dependent. The addition of the functional tool of CTP increased the sensitivity of CTA in the confirmation of brain death.

\section{REFERENCES}

1. Wijdicks EF, Varelas PN, Gronseth GS, et al. Evidence-based guideline update: determining brain death in adults-report of the Quality Standards Subcommittee of the American Academy of Neurology. Neurology 2010;74:1911-18

2. Wijdicks EF. The diagnosis of brain death. $N$ Engl $J$ Med 2001;344:1215-21

3. Wijdicks EF. Brain death worldwide: accepted fact but no global consensus in diagnostic criteria. Neurology 2002;58:20-25

4. Decree No. 96-1401 of December 2, 1996 concerning brain death diagnosis, organ, tissue, and cell harvesting for therapeutic or scientific uses and Circular D.G.S No. 96-733 of December 4, 1996. Official Journal of the French Republic; 1996

5. Dupas B, Gayet-Delacroix M, Villers D, et al. Diagnosis of brain death using two-phase spiral CT. AJNR Am J Neuroradiol 1998;19:641-47

6. Frampas E, Videcoq M, de Kerviler E, et al. CT angiography for brain death diagnosis. AJNR Am J Neuroradiol 2009;30:1566-70

7. Greer DM, Varelas PN, Haque S, et al. Variability of brain death determination guidelines in leading US neurologic institutions. Neurology 2008;70:284-89

8. Shemie SD, Doig C, Dickens B, et al. Severe brain injury to neurological determination of death: Canadian forum recommendations. CMAJ 2006;174:S1-13

9. Quesnel C, Fulgencio JP, Adrie C, et al. Limitations of computed tomographic angiography in the diagnosis of brain death. Intensive Care Med 2007;33:2129-35

10. Escudero D, Otero J, Marqués L, et al. Diagnosing brain death by CT perfusion and multislice CT angiography. Neurocrit Care 2009;11:261-71

11. Shankar JJ, Lum C, Sharma M. Whole-brain perfusion imaging with 320-MDCT scanner: reducing radiation dose by increasing sampling interval. AJR Am J Roentgenol 2010;195:1183-86

12. Shankar JJ, Lum C. Whole brain CT perfusion on a 320-slice CT scanner. Indian J Radiol Imaging 2011;21:209-14 